\title{
Mucho más allá del demo y el ensayo Estudio del alcance del programa Usinas Culturales
}

\author{
Far beyond demo and rehearsal. Study of the scope of \\ “Usinas Culturales" (culture factories) program
}

\section{Enrique da Rosa*}

\footnotetext{
* Diploma en Gestión Cultural, Facultad de Ciencias Sociales, Universidad de la República, Uruguay. Coordinador Departamental (Centros MEC), Ministerio de Educación y Cultura de Uruguay.

$\triangle \quad$ enriquedarosa@gmail.com https://orcid.org/0000-00019629-8048
}

\section{Resumen}

El Programa Usinas Culturales (UC) creado por el Ministerio de Educación y Cultura (MEC) tiene algunas señales de identidad propias del partido de gobierno que lo puso en marcha. Sin embargo, más allá de lo declarativo, la ejecución del programa tiene características particulares que en ciertos puntos no reflejan del todo los postulados anunciados en el programa del Frente Amplio.

Palabras clave: Estado, política cultural, gestión cultural, descentralización, Uruguay.

\begin{abstract}
The Cultural Factories Program launched by the Ministry of Education and Culture reflect the identity signs of the governing party that launched it. However, beyond declarative statements, the execution of the program has some distinctive characteristics that in certain aspects do not fully reflect the postulates announced in the Frente Amplio program.
\end{abstract}

Keywords: State, cultural policy, cultural management, decentralization, Uruguay. 


\section{Introducción}

El arribo de la izquierda uruguaya al gobierno generó una vasta instalación de proyectos, planes y programas enmarcados en el programa «progresista» que propuso en las elecciones de 2004. El llamado «barco insignia» de aquellas orientaciones fue el Ministerio de Desarrollo Social, creado desde cero para atender la emergencia social en una población empobrecida, desempleada, con escasa formación, con atención médica y vivienda deficientes. El novel ministerio coordinó, organizó y puso en práctica una serie de medidas para superar aquellas carencias, intentando acompañar a la población carenciada hacia la autonomía.

El camino elegido por el partido de gobierno fue el de llevar adelante fuertes políticas públicas que persiguieran el mismo objetivo y hacerlo desde el propio Estado, como forma de asegurar la continuidad de las políticas, fortaleciendo su viabilidad al dejarlas inscritas en ministerios y otros organismos estatales.

El camino elegido responde a una puntual interpretación del rol del Estado por la izquierda uruguaya. El partido de gobierno reconoce al Estado el papel de herramienta que debe liderar el desarrollo nacional y aportar a la democratización social, una posición explicitada en los documentos de su IV Congreso Extraordinario, de 2001:

En esta etapa el Estado deberá asumir, sin dudas, el liderazgo del desarrollo haciendo efectivo un concepto del cual nuestra fuerza política ha tomado conciencia tempranamente: el de la estrecha vinculación entre reformar el Estado y democratizar la Sociedad. (Frente Amplio, 2003)

Esta postura fue puesta en práctica en la salud, en la vivienda, en la educación, en las políticas sociales, en las laborales y en varias otras áreas y responsabilidades estatales.

Pero hay otro norte importante en las prácticas descritas, un posicionamiento político muy definido hacia la descentralización, sobre todo cuando se la piensa como vehículo de democratización del acceso a los bienes y servicios a toda la población.

En este artículo se abordará el caso específico de una política pública cultural que desde su planteo fundacional de gestión se enmarca entre estos pilares políticos, pero se queda por el camino: las Usinas Culturales.

\section{Re-wind}

A partir de 2009 el Estado, mediante la Dirección Nacional de Cultura, ha desarrollado el programa Usinas Culturales (UC), que consiste en la instalación y gestión de unidades 
tecnológicas equipadas con sala de grabación musical y tecnología para producción audiovisual.

Al día de hoy son 18 UC, 10 en la zona metropolitana y las restantes distribuidas de manera dispar en el interior del país. La mayoría se inauguró en el tramo que va de 2009 a 2014. En cada caso, fueron instaladas gracias a un acuerdo o convenio suscrito entre el Ministerio de Educación y Cultura (MEC) y otra contraparte, que en muchas oportunidades han sido los gobiernos departamentales.

\begin{tabular}{|l|l|l|}
\hline Usina cultural & Inauguración & Contrapartes \\
\hline Salto & Marzo de 2009 & Intendencia de Salto \\
\hline Comcar & Marzo de 2009 & Instituto Nacional de Rehabilitación \\
\hline Casavalle & Junio de 2009 & Intendencia de Montevideo \\
\hline Cerro & Octubre de 2009 & UDELAR-APEX \\
\hline Treinta y Tres & Octubre de 2009 & Intendencia de Treinta y Tres \\
\hline Castillos & Julio de 2010 & Intendencia de Rocha \\
\hline Carrasco Norte & Octubre de 2010 & Intendencias de Canelones y Montevideo \\
\hline Cerro Largo & Diciembre de 2010 & Intendencia de Cerro Largo \\
\hline Artigas & Noviembre de 2011 & Intendencia de Artigas \\
\hline Paysandú & Diciembre de 2011 & Intendencia de Paysandú \\
\hline Durazno & Enero de 2012 & Ministerio de Defensa \\
\hline San Carlos & Setiembre de 2012 & Intendencia de Maldonado \\
\hline Bella Italia & Agosto de 2013 & Intendencia de Montevideo y asociación civil \\
\hline Palermo & Setiembre de 2014 & Casa de la Cultura Afrouruguaya \\
\hline Parque Batlle & Octubre de 2014 & Intendencia de Montevideo \\
\hline Vilardebó & Noviembre de 2014 & ASSE \\
\hline Cordón & Mayo de 2016 & INJu-MIDES \\
\hline Peñarol & Mayo de 2017 & opP e Intendencia de Montevideo \\
\hline & & \\
\hline
\end{tabular}

Fuente: Elaboración propia con información obtenida en la web del MEC (s/f).

La web institucional del programa describe su objetivo como «promover el potencial creativo de la ciudadanía a partir del uso de las nuevas tecnologías» (Ministerio de 
Educación y Cultura [MEC], s/f), al tiempo que define su distribución territorial con base en lo que llama «lugares con un notorio déficit» de este tipo de servicios.

Hay casos puntuales que cabe destacar por su singularidad, como la instalada en la unidad 4 del Instituto Nacional de Rehabilitación Santiago Vázquez (antes COMCAR), una propuesta comprometida en la promoción de los derechos culturales de las personas privadas de libertad, como también de los funcionarios del lugar. Los recursos para esta UC se obtuvieron del Fondo para el Logro de los Objetivos del Milenio.

Otra experiencia de importancia es la desarrollada mediante la UC instalada en el Centro Diurno de Rehabilitación del Hospital Vilardebó, fruto del convenio firmado con la Dirección de Salud Mental de la Administración de Servicios de Salud del Estado (ASSE).

Los convenios firmados entre MEC y sus contrapartes determinan las responsabilidades y la inversión que cada parte compromete para el programa. En algunos casos esta inversión se traduce en la compra de materiales, instrumentos, equipos, el acondicionamiento edilicio, el mantenimiento de equipos, el pago de servicios del local y/o de recursos humanos, pero en con mayor frecuencia es el propio MEC el que carga con la inversión más importante, tanto en la compra de instrumentos como en el mantenimiento de la uc y en la contratación de recursos humanos.

El equipo humano de una UC está integrado por un técnico para la grabación de audio, un técnico audiovisual y un coordinador. Sin embargo, no todas las usinas cuentan con el equipo completo, en la mayoría de los casos por razones presupuestales, lo que determina que parte de los servicios no se brinde a la población.

\section{$\operatorname{Rec}$}

Muchas de las propuestas programáticas que el Frente Amplio presentó a la población rumbo a las elecciones se transformaron en acciones durante la gestión de gobierno. Es en esa gestión que las acciones son determinadas por puja de poderes en el entramado social, balance de fuerzas sociales, urgencias humanitarias, dependencias económicas, y sobre todo por convicciones ideológicas dentro del partido de gobierno. El resultado de estas transacciones terminó fijando la prioridad de las acciones, la velocidad de las reformas, la lentitud de las permanencias y la profundidad de los cambios.

Visto desde una perspectiva histórico-política, el Uruguay tuvo con el batllismo y el neobatllismo, en la primera mitad del siglo pasado, avances muy importantes en torno al redimensionamiento del Estado, que se hizo cargo de áreas hasta ese momento desatendidas. La propuesta que la izquierda desarrolla en su acción de gobierno retoma esta práctica política de fortalecer el Estado para desde él atender algunas de las necesidades de la población. 
Esto significa actualmente posicionar al Estado como un actor central, dejando atrás su desactualización, reducción y vaciamiento por las prácticas económicas de la dictadura y el envión neoliberal de los años noventa.

La gestión gubernamental actual plantea un Estado que sea motor de desarrollo y garante de derechos, que transparente su accionar y democratice espacios de poder. Para hacerlo plantea una variedad de programas que buscan optimizar la infraestructura ya existente, invertir en nueva y generar circulación de recursos humanos por los territorios que accedan a menos bienes y servicios, y define como estratégico el compromiso de los gobiernos departamentales como contrapartes ejecutantes de estas iniciativas.

En la puesta en marcha de este nuevo rol, el Estado se ha encontrado con varias trabas corporativas, especialmente cuando intenta recuperar algún área que había pasado a la gestión privada, como sucede, por ejemplo, con el transporte rutero de pasajeros y de carga, con los servicios de salud en el interior del país o con el mantenimiento de las redes de servicio privatizadas en tercerizaciones añejas.

Pero el Estado también ha venido disputando el espacio público para la iniciativa de acciones afirmativas. La importante cantidad de iniciativas legislativas de la llamada nueva agenda de derechos sigue encontrando escollos en una sociedad civil que se fragmentó en dictadura y se hiperindividualizó en los noventa, los años de la práctica neoliberal. La batería de leyes y proyectos de ley que han tenido por finalidad el amparo de la población desatendida sistemáticamente en lo que respecta a sus derechos personales suscita reacciones populares y corporativas inmediatas, en las que se advierten señas de una cultura con rasgos reaccionarios, homofóbicos, racistas, clasistas, antisindicales, egoístas, insolidarios y con radicalismos religiosos.

Según sus propios documentos internos, el partido de gobierno ha apostado a fortalecer la presencia del Estado, incluyendo la creación de nuevas estructuras, para brindar con calidad los servicios que la población necesita y para generar los espacios donde debatir con esa misma población el rumbo, la velocidad y la profundidad de sus planteos.

Esta postura de proactividad estatal necesariamente va acompañada de la descentralización, entendida como la creación de multiplicidad de espacios donde la población pueda, por un lado, recibir los bienes y servicios que le corresponden por derecho y, por otro, incidir (cada vez más) en las decisiones del Estado. Los gobiernos del Frente Amplio (nacional y departamentales) han puesto en marcha mecanismos de presupuestos participativos, llamados de fondos públicos mediante presentación de proyectos de desarrollo local, Consejo de Ministros descentralizado, mesas de desarrollo rural, consejos salariales por rama, promoción de la autogestión y soporte de empresas recuperadas por trabajadores que apuntan a esa participación social. 
Esta práctica difiere de la interpretación que antes se tenía en la administración pública sobre las características de la descentralización. En los años ochenta y noventa hubo una importante instalación de oficinas estatales en las capitales departamentales de todo el país, que amplió el área de cobertura de los servicios. Pero el planteo descentralizador de la izquierda en el gobierno se fundamenta en acercar el servicio territorialmente mucho más allá de las capitales y generar nuevas prácticas de gobierno directamente junto a la población que se quiere priorizar y promover.

La historia del desarrollo económico de Uruguay no siempre reflejó la mejor versión de la distribución de la riqueza; al contrario, se sustentó por mucho tiempo en la explotación de la población trabajadora y menos pudiente. Las propuestas de la fuerza política de gobierno van en el sentido contrario: el de un fortalecimiento individual y del entramado social referente a políticas sociales y políticas redistributivas. Y el Estado es planteado como herramienta para esto.

\section{Fast Forward}

La instalación de una nueva UC ronda hoy en día una inversión de cuatro millones de pesos aproximadamente, divididos entre obra y acondicionamiento edilicio, equipamiento y recursos humanos. Este monto de inversión debe generar el celo necesario para instalarla en el lugar de mayor provecho posible, dentro de los postulados del programa.

Como queda claro en el cuadro presentado anteriormente, las UC no tienen una lógica de distribución territorial; es más, últimamente contradicen su postulado de estar en territorios donde hay «notorio déficit» (MEC, s/f). La distribución no responde al criterio de priorizar departamentos por distancia de la capital, ni a los índices de menor desarrollo humano, ni a una selección de poblaciones de determinada cantidad de habitantes, por citar algunos criterios de distribución utilizados por otros ministerios para instalar sus inversiones. De las 18 UC, 10 están en el área metropolitana, y en el resto del país solo ocho departamentos cuentan con una.

Pero en octubre de 2014 y en mayo de 2016 hubo dos acciones concretas del programa que llaman la atención: en 2014 se instaló una UC en Parque Batlle y en 2016 otra en el Cordón, ambos barrios montevideanos céntricos, donde se concentra en altos porcentajes la población con mejores ingresos, con mayor proporción de formación terciaria y con la mayor concentración de oficinas de servicios públicos del país. Son zonas del país que están muy lejos de la definición de lugares objetivo de esta política pública.

Es cierto también que ambas están instaladas sobre nodos de las líneas de ómnibus de Montevideo, lo que facilita el acceso de la población periférica, ya que todos los 
recorridos llevan hacía allí, pero esta característica vial termina abonando a la centralidad y no a la descentralización.

Por otra parte, la decisión de instalar una nueva UC seguramente estuvo ligada a encontrar en uno de estos casos un socio, el Ministerio de Desarrollo Social (MIDES) Instituto Nacional de la Juventud (INJU), que tuvo cómo cubrir parte de esa inversión y ya tenía captados en el público de sus propias actividades el destinatario de las UC. No obstante, ambos organismos, MEC y MIDES, son de carácter nacional y deben velar por el acceso a los bienes y servicios de toda la población en todo el territorio, lo que queda cuestionado al no haberse instalado UC en las oficina del MIDES en el interior del país.

Cabe preguntarse cuál es el criterio real hoy en día para la instalación.

Para la redacción de este artículo se han realizado entrevistas telefónicas con técnicos de las UC en las que se puede observar que el perfil del usuario ha cambiado desde los primeros años del programa hasta hoy. Si en un principio el programa tuvo un usuario más cercano al perfil poblacional sin recursos y sin posibilidades de producir culturalmente por su situación de pobreza, fue pasando a una población de clase media, con más conocimiento musical y de producción. Hoy los técnicos describen situaciones en las que el perfil del usuario es más cercano a una clase media que no quiere pagar una sala de grabación, aunque tenga posibilidades económicas para hacerlo. Entonces las UC también son una propuesta de gratuidad dentro de un mercado de pago, aprovechado por toda la clientela.

No todas viven este cambio, sin embargo. Hay otras que permanecen trabajando con poblaciones de barrios como Casavalle o Carrasco Norte.

Entonces es plausible afirmar que cada usina trabaja con objetivos distintos, según el perfil de sus usuarios, en los recursos económicos de estos, en el conocimiento técnico y en el motivo que cada uno tiene para producir culturalmente.

Otros cambios generan aún más interrogantes, sobre todo el compromiso con el mantenimiento de las UC existentes y la nula instalación de nuevas en lugares con clara necesidad.

La experiencia que brindan los años de funcionamiento de este programa lleva a los técnicos a afirmar que el compromiso para instalar una usina no es el mismo que para mantenerla lo largo de los años. Las contrapartes no cumplen con sus aportes, la infraestructura sin mantenimiento se ve afectada con el paso del tiempo y es el MEC el que debe cubrir costos que en un principio eran el compromiso de otros. A su vez, la demora en reponer los equipos dañados hace que el servicio se vea cada vez más retrasado. En varios lugares el equipo de técnicos no está completo, por falta de presupuesto, por problemas contractuales y en buena parte por falta de voluntad política para resolver los problemas. 
Está claro que ninguna política pública se sostiene sin presupuesto; ninguna resiste mucho tiempo cuando los socios firmantes hacen caso omiso a sus compromisos o cuando no se la entiende como prioritaria por el gobierno local o nacional.

La falta de cumplimiento de lo acordado entre los gobiernos locales y nacionales hace que el Estado se encuentre con una importante traba en la división política del territorio, en el debate sobre las competencias de organismos territoriales y macroterritoriales, en la disputa de poder entre los partidos que gobiernan un departamento o un país, y al final el que fue considerado como un socio se transforma en un impedimento.

Estas disputas menores nunca son abiertamente declaradas, pero las acciones de los gobiernos departamentales dejan en claro un rumbo contrario al desarrollo de las políticas públicas. No solo ocurre con las UC; las actitudes de las intendencias departamentales de Florida y Tacuarembó con los Centros MEC, por ejemplo, muestran el mismo accionar, que los relega a espacios con menos infraestructura, en el primer caso, o los lleva al estancamiento, en el segundo.

Los habitantes de San Gregorio del Polanco y Achar, en el departamento de Tacuarembó, reunieron dos veces firmas para solicitar la instalación de Centros MEC en sus localidades, pero la Intendencia Departamental nunca aportó lo necesario para concretarlos.

En 2014 la Coordinación Departamental de Centros MEC de Florida denunciaba públicamente en la prensa departamental que la Intendencia trasladaba el Centro, sin la notificación correspondiente, a un lugar sin la infraestructura imprescindible. En entrevista televisiva decía que, por ser los Centros MEC «una política cultural de muchísimo éxito en el interior del departamento», probablemente había móviles políticos en el gobierno departamental para que no se la viera (Tv Florida Canal 4, 2014).

Más allá de abrir sus puertas a esta variada población con diferentes intereses, las UC no parecen estar conectadas a lineamientos centrales. Por más que trabajen sobre las especificidades de las realidades locales, no coordinan con las demás unidades del mismo programa. En varios casos en el interior del país la única coordinación se da entre la UC y la Dirección de Cultura departamental.

En este aspecto existe otra diferencia entre la teoría que dio lugar a los objetivos iniciales del programa y la práctica real de la política. Las direcciones departamentales de Cultura responden a las propuestas del gobierno departamental; esto no es ningún problema si las metas nacionales y departamentales coinciden, o por lo menos se acercan, pero cuando no es así la infraestructura y el patrimonio de la política pública pasan a responder a otra visión de trabajo.

Las dificultades que generan estas diferencias programáticas han sido planteadas en los debates departamentales del Plan Nacional de Cultura comenzado en 2016, que ya en su fundamento plantea: 
Porque el país precisa de una herramienta que permita pensar en términos de políticas públicas en cultura a largo plazo, que sirva para optimizar el sector en cuanto a recursos, acciones y mecanismos de trabajo. (MEC, 2015)

Por otra parte, el programa UC tiene una enorme dependencia de su socio-contraparte local para la instalación de nuevas unidades. Existen varias solicitudes de instalación en el interior del país a la espera de un socio que aporte lo faltante. Pero tampoco el MEC cuenta con una asignación presupuestal suficiente para instalar por lo menos una por año. El presupuesto anual del programa es de dos millones de pesos para todo destino, y la apertura de una UC tiene un costo aproximado de cuatro millones. La asignación presupuestal del programa imposibilita alcanzar su propio objetivo de desarrollar el potencial creativo de la ciudadanía.

Ante esta comprobación se hace evidente la necesidad de adecuar el discurso político respecto a la generación de nuevos programas y la viabilidad económico-financiera que implica su puesta en marcha y gestión cotidiana. Las urgencias sociales y culturales que la izquierda pretende solucionar exigen que sus políticas públicas cuenten con los recursos necesarios, que no dependan de las voluntades o coincidencias con gobiernos departamentales y que el surgimiento (o no) de socios no determine el crecimiento o el fracaso de esa política.

\section{Play}

Me detendré ahora específicamente en la producción musical desde las UC. Si el programa contara con las 18 unidades en funcionamiento con el equipo humano completo, en diálogo entre sí y acordes con los lineamientos centrales del programa, con un mantenimiento adecuado de equipos, aun así estaría lejos de desempeñar un papel estatal de relevancia.

Con un demo o un disco completo bajo el brazo, sin dar herramientas fuertes y actuales a los artistas, no se los ayuda a cambiar nada.

Las necesidades sociales y culturales que marcan los diagnósticos de los partidos políticos que promueven la superación de estas falencias deben ser enfrentadas con políticas públicas de metas claras y con objetivos que reflejen el compromiso político planteado. Para ser calificadas de coherentes, esas políticas deben marcarse metas alcanzables - sin caer en facilismos - y estar acompañadas de un esfuerzo presupuestal y político que les permita superar las falencias que ya se conocen en el territorio. Deben plantearse evaluaciones y replantearse alcances en tiempos cortos de gestión. 
La política pública cultural de producción musical como herramienta de cambio social necesita una red de UC en todo el territorio nacional que, atendiendo las características de cada territorio, desarrolle en conjunto los lineamientos centrales de promoción de los derechos culturales de toda la población, pero especialmente de la que menos recursos tiene.

Para cumplir sus objetivos declarados, esta red debe contar con un plan de formación musical coordinado con las escuelas de música de primaria, donde participe además la Escuela Universitaria de Música. Que el aprendizaje de un instrumento sea acompañado por la formación en la producción musical, en la preparación para el mercado, en el ensayo de combinar conocimientos con otros multiplicando el aprendizaje.

Parece a todas luces necesario que la política apunte a dos perfiles de usuario: por un lado, aquel que aún no considera a la música como un vehículo de identidad, de libre expresión y de creatividad basada en su realidad; por otro, el músico que no tiene recursos para presentarle a su público un primer trabajo con la seguridad necesaria.

Debe tener una propuesta que incluya la grabación del primer disco, la impresión de un número mínimo de copias, la colocación del trabajo en plataformas de acceso por internet (el programa actualmente ya cuenta con esto), la difusión de las grabaciones en espacios radiales de las emisoras del SODRE y en las radios comunitarias de onda compartida del MEC. Debe generar la circulación de los artistas por las ciudades de su región, reuniéndolos con músicos de otras UC, y garantizar la presencia en la capital del país, en escenarios públicos, con ciclos diferenciados por estilos musicales o por regiones geográficas. La política se acercaría más a sus objetivos declarados si lograra optimizar todos los recursos estatales, garantizara las alianzas necesarias y creara nuevas oportunidades para quienes toman la expresión artística como un camino de vínculo con la sociedad.

En Uruguay no existe un mercado discográfico hegemónico dirigido por disqueras multinacionales que impida el desarrollo de la producción nacional, pero hay una lógica de hipervisibilización de unos artistas en detrimento de otros. Una lógica de mercado que sostiene los mismos formatos desde hace mucho, incluso con nombres y apellidos que se eternizan. Que mayoritariamente genera circuitos de alcance metropolitano con músicos metropolitanos.

El éxito de las UC sería que buena parte de la población metropolitana conociera y disfrutara la producción musical de la otra mitad del país.

Incluso en los sectores vinculados con el mundo musical es imposible hoy, sin recurrir a Google, hacer un listado de cinco artistas riverenses, cinco artiguenses, cinco arachanes, cinco tacuaremboenses y así por delante. Es asombroso todo lo que los uruguayos se pierden de disfrutar. 
El registro actual y el rescate de registros anteriores de las expresiones musicales declaradas patrimonio inmaterial debe ser parte de esta política, una tarea para ser llevada adelante junto con la Comisión del Patrimonio Cultural de la Nación. Al igual que el papel importante que tienen el Archivo de la Voz del SODRE y el Centro Nacional de Documentación Musical Lauro Ayestarán, esta política debe sumarse a los esfuerzos por difundir el patrimonio sonoro de nuestro país.

En 2009 la UNESCO declaró al candombe como patrimonio cultural de la humanidad (Unesco, 2009), y en 2010, por decreto 414, el Poder Ejecutivo de Uruguay declaró patrimonio inmaterial del país el arte del payador, el toque de la llamada, la murga montevideana, el estilo, la milonga y el tango (decreto 414, 2010). Estas declaraciones patrimoniales necesitan políticas de registro y promoción, y las UC deben formar parte de estos planteos. En 2017 la Comisión del Patrimonio propuso a la Red de Directores de Cultura del país comenzar un largo trayecto de investigación y registro de los patrimonios inmateriales del interior del país. Es una oportunidad ideal para que un mejorado programa de Usinas Culturales cumpla un papel relevante en esta área.

Hay experiencias en América Latina que han marcado el camino en este tipo de registros. El proyecto América Afroindígena es uno de ellos. Con sede en la ciudad de México, este proyecto investiga, documenta, realiza producción fonográfica y audiovisual de las músicas afroindígenas de toda América, para difundirlas tanto en soportes tradicionales como en plataformas electrónicas (América Afroindígena, 2017).

Otros ejemplos de esta misma línea de trabajo son la Fonoteca Nacional de Chile especializada en el cancionero de cuecas chilenas (Fonoteca Nacional de Chile, s/f), Señal Memoria de Colombia, con una gran producción discográfica sobre música patrimonial (Radio Televisión Nacional Colombia [RTNC], s/f), y la Fonoteca Nacional de México, con una de las más completas colecciones de archivos históricos y patrimoniales en el campo sonoro (Fonoteca Nacional de México, 2017).

A su vez, proyectos nacionales como Mapa Sonoro de Uruguay - premiado por los Fondos Concursables-, llevado adelante por la antropóloga Ana Rodríguez, que desarrolla una fuerte labor de registro del mundo sonoro de los paisajes y personas del interior del país ("Mapa sonoro Uruguay", 2016), puede ser un gran socio en el reconocimiento de nuevos beneficiarios de una Usina Cultural democratizada.

Es evidente que las uc tienen mucho que aportarle al país y en especial a aquellos más alejados de los centros de distribución de bienes culturales. En este marco, es posible plantear la reformulación del programa en su totalidad para que pueda alcanzar el objetivo inicial de su creación, y se debe ser consciente de que si no se lo hace reconociendo la existencia de un mercado que no visibiliza lo que no domina, no se pueden dar pasos firmes con los futuros artistas de las UC. Estos pasos seguros que superen las lógicas de mercado deben tener una primera etapa de acompañamiento estatal de la evolución de la propuesta artística. Que no se entienda como control de un Estado censor, 
sino como la acción de poner a disposición las herramientas necesarias para fortalecer la propuesta.

Debe tener además una segunda etapa, de difusión y presentación del trabajo finalizado en corredores estatales. Al mismo tiempo se debe brindar formación en producción para los nuevos formatos de música, saliendo de los vinilos, los casetes e incluso de los CD y embarcando en plataformas digitales. Y aprovechar la gran gama de posibilidades que presentan la red Ceibal, la ANII o la pujante industria nacional del software. Todos ellos son caminos que ya las disqueras de mercado e independientes utilizan hace algún tiempo.

Y una tercera etapa que proponga la autogestión, el despegarse de la órbita del Estado y pasar a producir de manera independiente. El ecosistema de sellos uruguayos presenta hoy una rica diversidad de formatos organizativos; sellos que sostienen estilos musicales puntuales, que promueven la especialización de etapas de posproducción y se conectan directamente con escenarios internacionales.

Este nuevo modelo de usina debe dejar en claro la situación de los derechos de autor de los usuarios y velar por los derechos de los autores, pero también promover la producción musical libre de derechos de autor o con derechos comunitarios.

\section{Stop}

El Estado debe asegurar la ejecución de sus políticas públicas cuando son afectadas por la irresponsabilidad de los socios en los acuerdos firmados, pero también debe zanjar la utilidad de la libre interpretación que hacen los gobiernos departamentales de sus competencias. Si las políticas públicas son las acciones que el Estado lleva adelante y si los gobiernos departamentales son parte del Estado, estos no pueden huir a la responsabilidad de sostener el cumplimiento de las políticas públicas.

La falta de compromiso de los socios locales con las políticas nacionales se ha convertido en la principal debilidad de programa de Usinas Culturales. Es entonces imprescindible buscar maneras de disminuir el riesgo de abandono e imaginar formas de no caer en la trampa del «único socio disponible» o de la «autonomía departamental».

Intentaré ilustrar con un ejemplo de otro ámbito la tensión entre las disposiciones nacionales y su aplicación en el terreno departamental. El uso del casco es obligatorio por ley nacional y está demostrado que evita la muerte de motociclistas. Ante esta legislación nacional y las políticas de salud y cuidado del tránsito, no cabe ninguna posibilidad de que un gobierno departamental se oponga a la obligatoriedad argumentando cualquier cosa referente a la costumbre local de no usarlo. 
Las UC no son cascos que eviten muertes, pero son un programa implantado por el Estado uruguayo dentro de las acciones de un gobierno nacional. Los gobiernos departamentales no pueden torcer el objetivo del programa ni mostrarse omisos a su instalación y aplicación.

Este planteo obviamente generará profundos debates entre esferas de gobierno, que se disputarán la autonomía de sus acciones bajo el supuesto riesgo de intervención del Estado central en las definiciones de los ámbitos departamentales. Mientras dure esta puja seguirá congelado el avance de los derechos culturales de los ciudadanos. Frente a esto será preciso que el Estado se replantee los acuerdos locales, buscando la contraparte en otro organismo y preferiblemente en una organización social con anclaje en la tarea territorial. Habrá que analizar si los debutantes Puntos de Cultura convocados por la Dirección Nacional de Cultura no pueden cumplir este rol.

A lo largo de la gestión del Frente Amplio, las políticas sociales han ido fortaleciendo organizaciones sociales, instituciones territoriales en la periferia de Montevideo y el interior del país, con las que el Estado ha compartido tareas. Son ejemplo de ello los acuerdos firmados con ONG, sindicatos, gremios de profesionales, cooperativas sociales y cooperativa de artistas, en órbitas tan variadas como la salud, la cultura, la formación para el trabajo, la asistencia en políticas sociales y la promoción del cuidado del medio ambiente. Este es un antecedente importante para una posible acción del Estado que se centre en reconocerles a estas organizaciones el papel de socias-contrapartes en la ejecución de las políticas públicas.

La importancia de asegurar la viabilidad de las políticas estatales no es menor desde el punto de vista administrativo, porque encierra el mantenimiento de las relaciones democráticas y el derecho ciudadano de acceder a los bienes y servicios. El conflicto por este acceso se da entre gobiernos nacionales y departamentales, pero también frente a las lógicas de mercado.

El planteo de la izquierda uruguaya de un Estado garante de derechos y que actúe como promotor del desarrollo se encuentra de frente con otras posturas políticas que reclaman una disminución del Estado y denuncian a las políticas sociales como acciones paternalistas. Estas críticas son parte de una visión política y partidaria, pero también se sostienen en el discurso de una importante parte del empresariado uruguayo que se siente afectado cuando el Estado recupera parte de sus atribuciones o ingresa en un área que hasta ese momento era únicamente definida y ejecutada por intereses privados. Muestra de ello es la demanda que la Cámara de Industrias de Uruguay presentó en la Organización Internacional del Trabajo contra el sistema de negociación colectiva aplicado en los últimos años en el país, que cambió radicalmente las situaciones de sofocamiento de los trabajadores al momento de negociar pautas salariales.

Dentro de la lógica del partido de gobierno, anunciada en sus documentos, cada vez que los derechos sociales y culturales inalienables a las personas son cotizados 
como emprendimientos comerciales puntuales que compiten en un mercado desigual, el Estado debe cumplir un papel. Incluso en las resoluciones del IV Congreso del Frente Amplio (2003), en dos de sus apartados (Políticas sociales y Salud), se señala el rechazo a los proyectos de mercantilización de la educación impulsados por la OMC y se denuncia la penetración de la mercantilización en la atención sanitaria, ante lo que se propone reforzar el rol del Estado en defensa de estos derechos entendidos como esenciales.

Las industrias culturales, como la discográfica, cuando solo miden su éxito en avances cuantitativos en un mercado caracterizado por volátiles momentos de ventas marcados por modas, dejan de lado la importancia del proceso colectivo de elaboración del contenido cultural.

Incluso el actual partido de gobierno reconoce que la cultura por sí sola no generará cambios estructurales que aproximen democráticamente a una nueva sociedad, pero que por su propia naturaleza de espacio de lucha podrá generar el comienzo de los debates sobre esos cambios. Porque, además, ningún cambio estructural de la sociedad se asentará si no existen cambios culturales que lo determinen y antecedan, generando su base social. La cultura deja de ser lo decorativo y pasar a ser lo operativo del cambio social.

La gestión cultural (y sobre todo la gestión cultural estatal) significa trabajar sobre los cambios culturales internándose en ámbitos supuestamente no culturales en una primera acepción, incluida la lucha por superar lógicas del mercado. Víctor Vich lo dice de manera más clara en Desculturizar la cultura:

Las políticas culturales (y la gestión derivada de ellas) deben ser entendidas como una opción para hacer más visible la centralidad que tienen los significados (vale decir, los procesos simbólicos, los objetos culturales) en la estructuración del mundo social y para intentar salir de las ideologías que lo sostienen. En ese sentido, las políticas culturales deben partir por cuestionar aquella ideología que entiende el progreso social como la pura acumulación económica o como el fortalecimiento exclusivo del individuo liberal. Se trata, por el contrario, de gestionar la cultura a fin de posicionarla como un recurso crítico para construir mayor ciudadanía. A través de la introducción de nuevas representaciones de las identidades existentes, a partir de hacer más visibles los antagonismos sociales y de presentar nuevas formas de imaginar la vida, las políticas culturales pueden comenzar a construir ciudadanos más justos y más involucrados en el bien común. (Vich, 2014, p. 18) 


\section{Referencias bibliográficas}

AmÉRICA AFroindígEnA (2017). América afroindígena. Recuperado de www. americafroindigena.org.

Decreto 414 (2010). Modificaciones al reglamento de licencias de telecomunicaciones. Recuperado de https://www.impo.com.uy/bases/decretos/414-2010.

Ministerio de EduCACión y Cultura (s/f). Programa Usinas Culturales. Recuperado de: http://cultura.mec.gub.uy/innovaportal/v/35412/8/mecweb/programa?3colid $=3584 \&$ breadid $=35329$.

Ministerio de EdUCACIÓn y CULTURA (2015). Documento base hacia el Plan Nacional de Cultura «Dr. Gonzalo Carámbula». Montevideo: MEC.

Fonoteca Nacional De Chile (s/f). Fonoteca Nacional de Chile. Recuperado de http://fonotecanacional.cl/.

Fonoteca Nacional de México (2017). Fonoteca Nacional de México. Recuperado de http://www.fonotecanacional.gob.mx/index.php/fonoteca-nacional/presentacion.

FRENTE AMPLIO (2003). Grandes lineamientos programáticos para el gobierno 2005-2009: IV Congreso Extraordinario del Frente Amplio. Montevideo: Frente Amplio.

Mapa Sonoro de Uruguay (2016). Recuperado de www.mapasonoro.uy.

SeÑAl Memoria (s/f). Señal Memoria. Recuperado de http://www.senalmemoria.co/. TV FLORIDA CANAL 4 (2014). Lo políticamente cultural: Entrevista a coordinador de Centros MEC. Recuperado de http://www.tvflorida.com.uy/?p=6091.

UNESCO (2009). Tango y candombe declarados patrimonio cultural intangible de la UNESCO. Recuperado de https://news.un.org/es/story/2009/09/1175141.

VICH, V. (2014). Desculturalizar la cultura: La gestión cultural como forma de acción política. Buenos Aires: Siglo XXI. 